\title{
Range of Motion After Total Hip Arthroplasty: Simulation of Non-axisymmetric Implants
}

\author{
Constantinos Nikou ${ }^{1}$, Branislav Jaramaz ${ }^{1,2}$, and Anthony M. DiGioia ${ }^{1,2}$ \\ 1 Center for Medical Robotics and Computer Assisted Surgery, \\ Carnegie Mellon University, Pittsburgh, PA \\ costa@ri.cmu.edu http://www.mrcas.ri.cmu.edu \\ 2 Center for Orthopaedic Research, UPMC Shadyside Hospital, Pittsburgh, PA \\ \{branko, digioia\}ocs.cmu.edu http://www.cor.ssh.edu
}

\begin{abstract}
Dislocation following total hip replacement surgery represents a significant cause of early failure, incurring additional medical costs and patient distress. One major cause of dislocation is implant impingement. The most important factor in preventing implant impingement is correct implant orientation. This paper describes the newest version of a prosthetic range of motion simulator which permits prediction of prosthetic range of motion for non-axisymmetric femoral and acetabular implants. This analytical methodology could be used as a preoperative simulation tool that can help surgeons decide the implant placement that reduces the chance of implant impingement. Coupled with a computerassisted clinical system for precise implant positioning, this approach could significantly reduce the risk of dislocation, maximize the "safe" range of motion, and minimize the risk for complications arising from impingement.
\end{abstract}

\section{Introduction}

The incidence of implant dislocation following total hip replacement (THR) surgery ranges between 2 and $6 \%[3,12]$ and represents a significant cause of early implant failure, incurring additional costs to the total surgery expenses. The causes of dislocation are related to factors such as surgical approach, soft tissue tension, prosthetic design, and most importantly, orientation of components. One possible dislocation mechanism is the impingement of prosthetic components, in which the implant neck hits the rim of the acetabular liner and levers the head out of the socket [2]. In addition to dislocation, implant impingement causes excessive wear of the cup liner and creation of debris. Proper alignment of implant components, which is patient and implant specific, reduces the incidence of impingement and reduces the risk of associated complications.

Previously, we have developed and experimentally verified a preoperative analytical simulator that takes into account implant design, placement and orientation, and predicts the range of motion (ROM) and impingement limits [5, 6]. The simulator enabled surgeons to preoperatively optimize the parameters in the placement of axisymmetric implants in order to reduce the probability 
of implant impingement and dislocation during normal patient motion. However, non-axisymmetric cup liners and femoral implant necks, provided as part of commercial pre-designed implant systems and as custom implants, are in wide use. Recognizing this, we have extended the prosthetic range of motion (PROM) simulator to include models of non-axisymmetric implants.

Coupled with CT-based three-dimensional preoperative planning and imageguided positioning of implant components, this methodology has the potential of precise implementation, ensuring optimal outcomes with respect to risk of dislocation.

\section{Background}

The most common cause of dislocation after THR is implant impingement caused by malposition of components [12]. A number of researchers and clinicians have examined this phenomenon, in an effort to explain mechanisms of dislocation. Amstutz and Markolf [2] described three modes of dislocation: 1) due to poor tissue tension, the prosthetic head climbs the socket wall and slips over the rim of the socket, 2) the neck impinges on the socket wall and levers the head from the socket, and 3) the neck impinges on a bony prominence.

Some researchers have tried to identify the range of cup orientations that are less prone to dislocations, based on the geometric similarity of human anatomies. Lewinnek et al. [10] demonstrated that the cases falling in the zone of $15 \pm 10$ degrees of anteversion and $40 \pm 10$ degrees of abduction have an instability rate of $1.5 \%$, compared with a $6 \%$ instability rate for the cases falling outside this zone. No attention was paid, however, to the design of either the cup, the design of the femoral component, or the femoral component orientation.

Although the mechanism of implant impingement is well understood, the attempts to model the phenomenon were until recently limited mainly to experimental procedures, in which a physical model is created to simulate the range of motion. Most investigators $[2,12]$ realized that the head-to-neck ratio of the femoral component is the key factor of the implant impingement. However, few have attempted to quantify the relationship between the implant design and orientation and the incidence of dislocation. Some experimental studies examined how specific implant design influences the prosthetic range of motion. Amstutz et al. [1] and Krushell et al. [8] examined experimentally the influence of different prosthetic designs and the influence of prosthetic orientation. However, general quantitative results based on constant head-neck ratios cannot be readily applied to use of non-axisymmetric implants because the head-neck ratio for an implant pair changes as a function of implant and position.

Other researchers have examined the effect of acetabular cup design. Krushell et al. [9] evaluated the ROM of two types of elevated-rim liners compared with standard liners. They concluded that an optimally oriented elevated-rim liner may improve the joint stability with respect to implant impingement. Cobb et al. [3] have demonstrated a statistically significant reduction of dislocations in the case of elevated-rim liners, compared to standard liners. The two-year probability 
of dislocation was $2.19 \%$ for the elevated liner, compared with $3.85 \%$ for the standard liner. They raised the concern, however, of possible long-term effects of the elevated liner on wear and loosening. Initial results of a finite element study by Maxian et al. [11] indicate that the contact stresses and therefore the polyethylene wear are not significantly increased in the extended lip case.

Analytical modeling of range of motion has only recently become a subject of interest for researchers. Maxian et al. [11] have looked at the dislocation propensity for different liner designs, although dislocation was not considered in the context of range of motion. Jaramaz et al. developed an analytical model (along with experimental validation) for calculating range of motion for a given size and orientation of only axisymmetric implant components $[5,6]$.

\section{Methods}

\subsection{Coordinate systems and transformations}

In order to establish appropriate relationships between the leg motion, position of the pelvis in the body, and relative orientations of implant components, we have to define relevant coordinate systems. We define the body coordinate system as follows: the $\mathrm{Y}$ axis points superiorly, parallel to the coronal and midsagittal planes; the $\mathrm{X}$ axis points to the patient's left, parallel to the coronal and transversal body planes; the $\mathrm{Z}$ axis is perpendicular to both $\mathrm{X}$ and $\mathrm{Y}$ and points to the anterior (Figure 1).
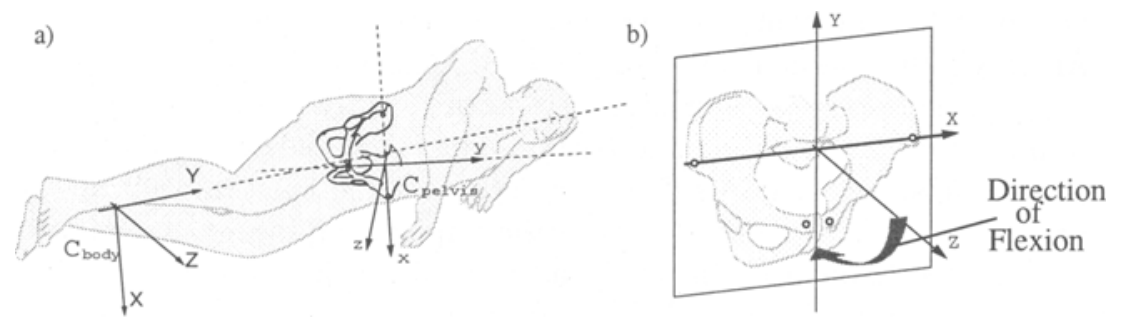

Fig. 1. a) Body and pelvic coordinate systems. b) Definition of the pelvic coordinate system as defined by landmark points on the pelvis

To define the pelvic coordinate system we use the pelvic anterior plane, defined by the anterior superior iliac spine and pubis symphisis points, and the symmetry plane of the pelvis (Figure 1). The pelvic $\mathrm{X}$ and $\mathrm{Y}$ axes lie parallel to this plane, with $\mathrm{X}$ to the patient's left, and $\mathrm{Y}$ pointed toward the patients head. Position of the pelvis is expressed as a flexion value. We define flexion as rotation about the body $\mathrm{X}$ axis. Neutral pelvic position (where the pelvis has zero degrees flexion) is defined as being aligned with the body coordinate system. The 
orientation of the pelvis relative to the body can be expressed as a $3 \times 3$ rotation matrix body $\mathbf{R}_{\text {pelvis. }}$. This transform, along with its inverse, ${ }^{\text {pelvis }} \mathbf{R}_{b o d y}$, can be easily derived from the flexion value.

Furthermore, we define a femoral coordinate system so that one axis (Y) corresponds to the mechanical axis of the femur, i.e., connects the center of the femoral head and the center of the condyles. The other axes are parallel with corresponding body axes in neutral standing position. Following common definitions, flexion/extension is then defined as rotation around the body $\mathrm{X}$ axis, $\mathrm{ab} /$ adduction as rotation around the body $\mathrm{Z}$ axis, and internal/external rotation as rotation around the femoral $\mathrm{Y}$ axis. $\mathrm{Ab} /$ adduction in flexion is rotation around the body Y axis subsequent to flexion. Any motion and position of the leg can be described as a result of a sequence of these basic motion components. We can establish a $3 \mathrm{D}$ rotation matrix $b^{\circ} y_{\mathrm{R}} \mathrm{femur}_{\mathrm{r}}$ which describes the position of the femur relative to the body frame. Femoral neutral position is attained when the femoral coordinate frame is aligned rotationally with the body frame.

Both the femoral implant neck and the acetabular cup have associated coordinate systems as well. The orientation of the femoral implant neck is described as an ordered pair of abduction and anteversion rotations relative to the femoral coordinate frame. Neck abduction is defined as rotation about the femoral Z (the coordinate axis pointing to the anterior) and neck anteversion is rotation about the femoral Y. Again, these rotations are sequential, with abduction occurring first. The rotations from the neutral position define a $3 \mathrm{D}$ rotation matrix femur $_{\text {neck. }}$.

The coordinate frame of the cup is defined as follows: the origin lies at the center of femoral rotation; the $\mathrm{Z}$ axis (also referred to as the cup axis) is normal to the opening of the cup; the other axes are normal to the $\mathrm{Z}$ axis. Normally, cup placement is described as a pair of rotations. DiGioia et al. described three systems for describing cup placement [4]. Their studies, however, assumed an axisymmetric cup, so only two parameters were required to describe cup orientation. The current PROM simulator supports these three description systems appending a rotation about the cup $\mathrm{Z}$ axis (a cup "twist") to each. Any of these systems is sufficient to define a full $3 \mathrm{D}$ rotation in the pelvic frame ${ }^{\text {pelvis }} \mathbf{R}_{\text {cup }}$, along with its inverse ${ }^{c u p} \mathrm{R}_{\text {pelvis. }}$. By the rules of transform arithmetic, we can calculate the position of the implant neck in the cup coordinate frame by the equation:

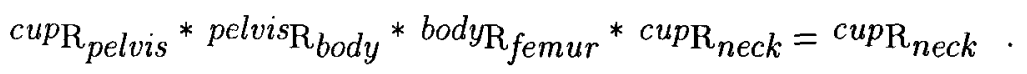

\subsection{Displaying range of motion}

The parameters necessary to evaluate PROM limited by neck-liner impingement are the geometries of both the implant neck and the cup liner, and the orientation of the neck relative to the cup implant liner. Leg motion is defined as motion relative to the body coordinate system. It is important to define leg motion to be relative to the body and independent of pelvic position because studies have 
shown significant differences in pelvic orientation for various patient positions (e.g., sitting vs. standing positions) [7].

a)

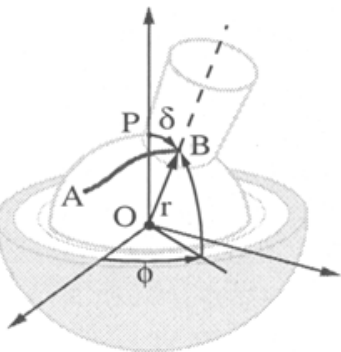

b)

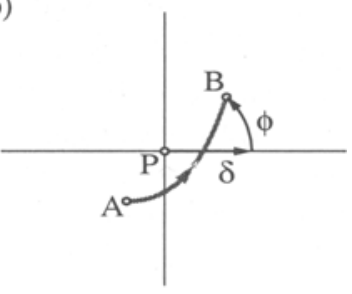

Fig. 2. Definition of angles $\phi$ and $\delta$ and their corresponding value on the PROM plot. Movement of the neck axis from point A to point B (diagram "a") corresponds to curve $\mathrm{AB}$ on the PROM plot (diagram " $\mathrm{b}$ ")

The knowledge of these parameters allows us to analyze the motion of the femoral implant neck in the coordinate frame of the acetabular cup. In a special case of the axisymmetric femoral neck, the problem can be reduced to tracking the axis of the neck liner and detecting whether it is within a domain defined by the cup liner geometry. For any position of the leg during motion one can track the position of the femoral neck in the spherical coordinate system placed in the center of the femoral head. These positions can be simply displayed on a two-dimensional polar coordinate plot as a function of two angles, $\phi$ and $\delta$ (Figure 2). The permissible ROM domain of the cup for a given neck geometry can then be defined by an "impingement circle" (this object is not necessarily circular, but a closed curve in $(\phi, \delta)$ space) where the limits of motion are plotted in the same $(\phi, \delta)$ space as the neck trajectory [5].

For more general implant geometries, such a simplification is not possible. In the non-axisymmetric case, the safe PROM will change for a given cup/femoral implant pair depending on femoral orientation. The safe ROM envelope is therefore not independent of neck orientation as in the axisymmetric case because the limits in any direction are dependent on the point of the neck where impingement will occur (Figure 3). In order to keep analysis simple, however, a two-dimensional ROM plot is still desired.

The rim of the cup liner is represented on the plot as a closed curve in $(\phi, \delta)$ space. This curve also represents the envelope of safe (non-impinging) PROM. The trajectory of the implant neck can be displayed as the path of its axis in $(\phi$, $\delta)$ cup space. Any trajectory that maintains safe PROM will lie within the cup liner curve. The neck trajectory curve represents only the path of the neck axis, however. We need to represent the surface of the neck because that is what will collide with the cup liner. 
a)

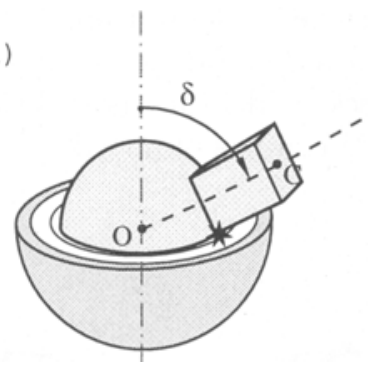

b)

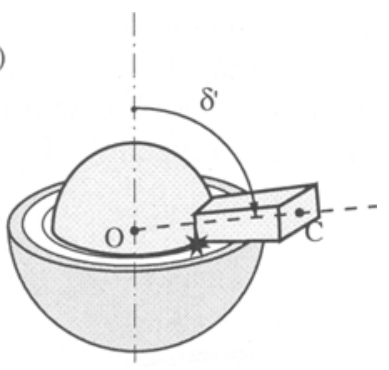

Fig. 3. Dependency of maximum $\delta$ angle on rotation orientation of the femoral implant neck. PROM is much greater in certain directions for case " $a$ " than case " $b$ "

When the neck of the implant impinges, it most likely impinges at one point only. This point lies on the cup liner as well as the neck. This point lies on a sphere centered at the origin $O$. The intersection of this sphere with the femoral neck surface is a closed curve which can be plotted on the PROM plot in $(\phi, \delta)$ coordinates. The impingement point lies on the plot at the intersection of the cup liner curve and the neck outline (Figure 4).

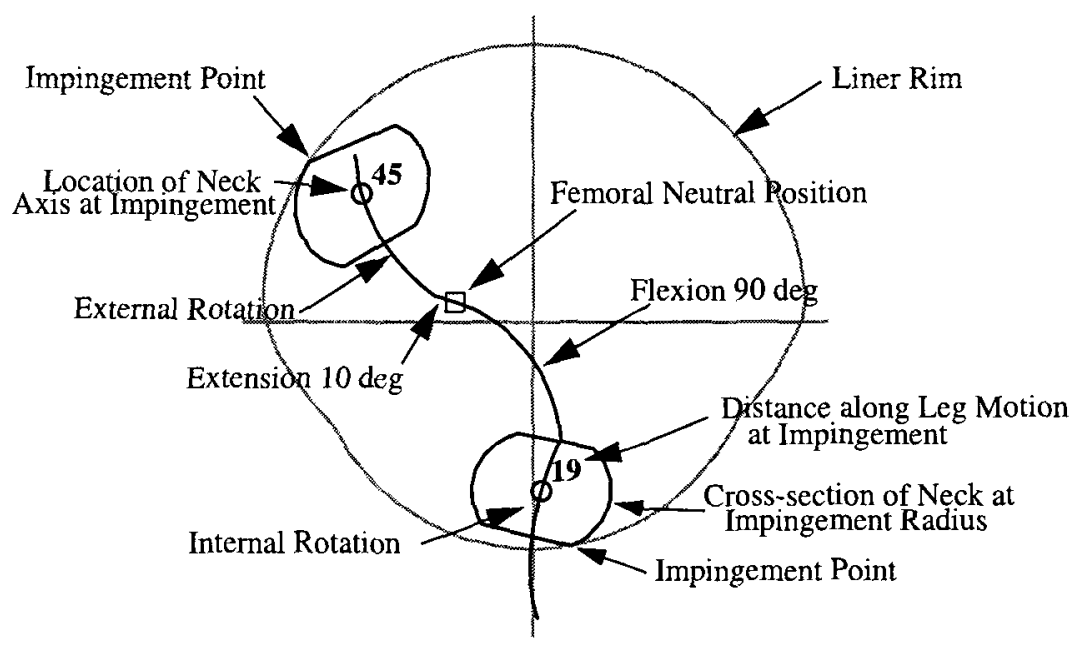

Fig. 4. PROM Plot example

\subsection{Modeling and collision detection}

The cup liner model is defined by a collection of ordered segments. These segments are a discretization of a continuous curve on the edge of the cup liner 
where impingement will occur. Inherent in the model is a point on the negative cup axis. Each segment on the cup along with this common point defines a triangle (see Figure 5). If any section of the neck model (discussed below) intersects one of these "cup polygons", then impingement has occurred.

a)

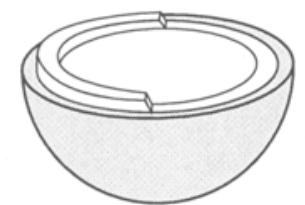

b)

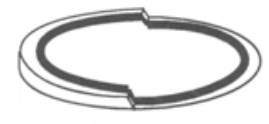

c)

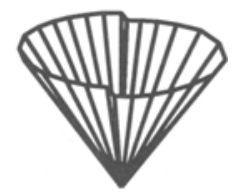

Fig. 5. Diagrams showing a) the cup with liner, b) the liner with highlighted rim, and c) the generated model of the cup

The femoral neck is modeled as a set of segments that is a discretization of the surface of the implant neck. During collision detection, only the neck segments that lie in the same range of distance from the center of rotation as the segments defining the cup liner need to be included in testing. Therefore, the runtime neck model generation can ignore all irrelevant sections of a large femoral implant surface model. This allows full implant data (e.g. a polygonal mesh of the implant surface) to be easily included in PROM analysis with minimal model editing. (In the case of the polygonal mesh, a simple script converting polygon data to simple segment data could be quickly run.)

Impingement limits are detected by moving the leg in small steps along the desired motion path. The size of the steps is determined by desired accuracy. The leg is moved incrementally until collision is detected. With complicated models, however, the angle limits become a function of not only the models, but also the position of the implants. Determination of impingement at each incremental step along the desired test path with this approach is too computationally intensive to be done interactively. Interactive speed is desired in the long run because the PROM simulator may eventually be extended to provide intra-surgical planning capabilities in computer-aided hip replacement.

In order to gain speed while retaining accuracy, a binary searching method can be implemented. For each leg motion path (divided into independent movements of flexion, abduction, extension, etc.), the full movement is first tried. If no impingement occurs during the movement, then the next movement is considered. Full movements are attempted until there are no more movements to try, or until impingement is detected. For the movement in which impingement is detected, movements of decreasing distance along the path are attempted until impingement occurs. The incremental distance attempted is halved each time, hence the term "binary search". When the incremental distance needed for impingement falls below a pre-set threshold, the position of the leg at this 
impingement point can be recorded. Below is pseudocode for the impingement detection algorithm:

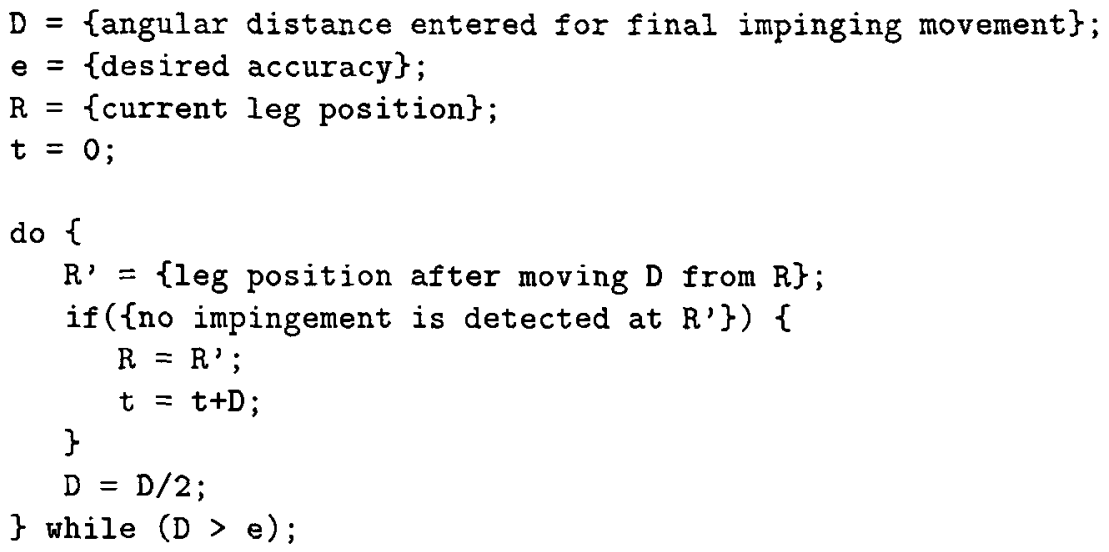

At this point, $t$ is the distance travelled along the movement path before impingement.

\section{Results}

Various simulations were performed with combinations of the Trilogy line of acetabular cup implants and VerSys femoral stem implants (both by Zimmer Inc.). A head size of $28 \mathrm{~mm}$ was assumed for all tests as well as a stem size of 17 . Work is currently being done to develop a database of implant data. Unless otherwise noted, all examples used an identical cup orientation $\left(45^{\circ}\right.$ abduction, $15^{\circ}$ flexion, and $135^{\circ}$ clockwise twist), an identical neck orientation $\left(40^{\circ}\right.$ abduction, $15^{\circ}$ anteversion) and no pelvic flexion.
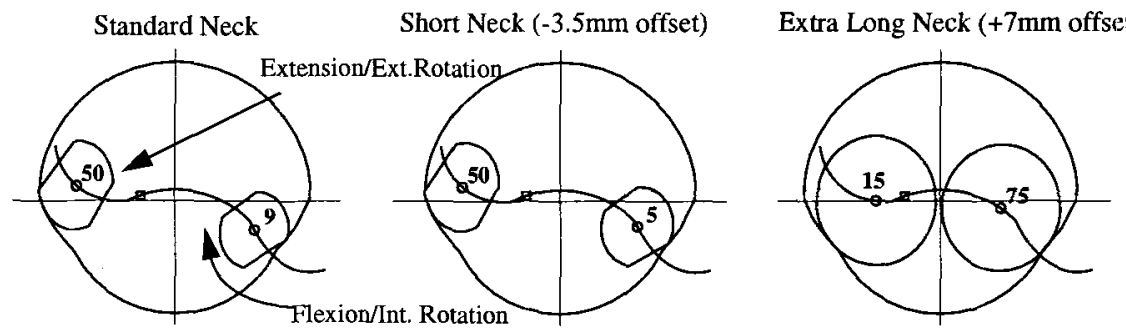

Fig. 6. PROM Plots for identical leg motions, but for different neck lengths

The first example illustrates the effect of neck length on PROM for the VerSys 20 degree elevated cup liner. In Figure 6, we see two sets of motions: 1) $90^{\circ} \mathrm{leg}$ flexion followed by maximum internal rotation and 2) $10^{\circ}$ extension followed by 
maximum external rotation. The PROM is least limited by use of the standard neck length, though use of a shorter neck does not shrink the envelope of possible motion by much. However, use of the $+7 \mathrm{~mm}$ neck extension results in a loss of $35^{\circ}$ possible external rotation. Flexion is limited to $75^{\circ}$ with no possible internal rotation. Notice the dramatically increased cross-sectional area of the effective neck for the extra long neck case, resulting from the "flange" on the long neck.

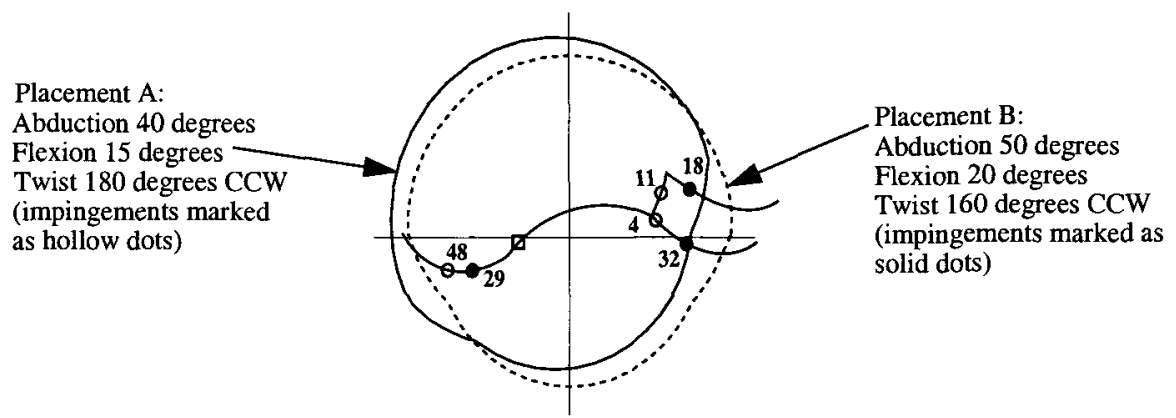

Fig.7. PROM Plots for different placements of the cup and liner

The second example of the PROM simulator reiterates the importance of proper placement of the cup during THR. Figure 7 shows a set of motions (the previous two motions along with $90^{\circ}$ flexion followed by $20^{\circ}$ adduction and maximum internal rotation) for two different cup placements. The PROM simulator allows the surgeon to superimpose two cup placements for comparative purposes. (The neck outlines have been removed to aid reading of the plots.) Although both cup orientations are within a commonly accepted "safe" range [10], Placement A will allow $6^{\circ}$ more external rotation, while Placement $\mathrm{B}$ provides a greater range of motion in the direction of forward leg motion, providing 11 to 14 degrees more internal rotation.

\section{Discussion}

Non-axisymmetric implants are used to increase implant stability and improve patient range of motion without compromising the strength of the implant. However, use of these implants increases the need for both proper selection and proper placement of the implant. Use of analytical simulators for implant design can allow suppliers to perform "trial runs" on their implants in order to fine tune their designs. They can allow surgeons to provide better care for their patients by placing the implants in order to optimize ROM on a per patient basis. Our studies along with others have shown that even within a widely-accepted "safe" zone, patient range of motion limited by impingement is affected significantly by cup placement. With the growing variety of available implant designs, some method 
of accurately performing optimally planned implant placement is increasingly important.

Though the current PROM simulator may predict impingement for various fixed positions of the pelvis, our results do not take into account motion of the pelvis during leg motion. Pelvic motion during movement will allow range of motion greater than the limits predicted by our system, although the extent of the allowance has yet to be determined. This system currently does not address dislocation via bone impingement. However, the complications that arise from implant impingement may be avoided with the proper combination of preoperative planning and technologically enhanced surgical procedures.

\section{References}

1. Amstutz, H. C., Lodwig, R. M., Schurman, D. J. and Hodgson, A. G.: Range of motion studies for total hip replacements. Clinical Orthopaedics and Related Research (111), September 1975, 124-130.

2. Amstutz, H. C. and Markolf, K. L.: Design features in total hip replacement. In Harris W.H. (ed.): Proceedings of the Second Open Scientific Meeting of the Hip Society, New York, C.V. Mosby, 1974.

3. Cobb, T. K., Morrey B. F., Ilstrup D. M.: The elevated-rim acetabular liner in total hip arthroplasty: Relationship to postoperative dislocation. The Journal of Bone and Joint Surgery, Vol 78-A, No. 1, January 1996, 80-86.

4. DiGioia III, A. M. et al.: An image guided surgical navigation system for the accurate measurement and alignment of acetabular implants. Accepted for publication: Clinical Orthopaedics and Related Research, 1998.

5. Jaramaz, B., Nikou, C., DiGioia III, A. M.: Effect of cup orientation and neck length in range of motion simulation. 43rd Annual Meeting, Orthopaedic Research Society, February 9-13, 1997, San Francisco, California, 286-48.

6. Jaramaz, B., Nikou, C., Simon D. A., DiGioia III, A. M.: Range of motion after total hip arthroplasty: Experimental verification of the analytical simulator. In Troccaz, J., Grimson, E., Mosges, R. (eds.): Proceedings of CVRMed-MRCAS97, Grenoble, France, March 1997: 573-582.

7. Johnson, R. C., and Smidt, G. L: Hip motion measurements for selected activities of daily living. Clinical Orthopaedics and Related Research 1970 Sept-Oct (72): 205-215.

8. Krushell, R. J., Burke, D. W., and Harris, W. H.: Range of motion in contemporary total hip arthroplasy (the impact of modular head-neck components). The Journal of Arthroplasty Vol. 6 (2 1991): 97-101.

9. Krushell, R. J., Burke, D. W., and Harris, W. H.: Elevated-rim acetabular components: Effect on range of motion and stability in total hip arthroplasty. The Journal of Arthroplasty 6, Supplement October 1991: 1-6.

10. Lewinnek, G. E., Lewis, J. L., Tarr R., Compere, C. L., Zimmerman, J. R.: Dislocation after total hip-replacement arthroplasties. J. Bone Joint Surg.: 217-220, Vol 60-A, No. 2, March 1978.

11. Maxian, T. A., Brown, T. D., Pedersen, D. R. and Callaghan, J. J.: Finite element modeling of dislocation propensity in total hip arthroplasty. 42nd Annual meeting, Orthopaedic Research Society, February 19-22, 1996, Atlanta, Georgia, 259-44.

12. McCollum, D. E. and Gray, W. J.: Dislocation after total hip arthroplasty (causes and prevention). Clinical Orthopaedics and Related Research 261 (1990): 159-170. 